\title{
Fast Global Minimization of the Chan-Vese Model for Image Segmentation Problem
}

\author{
Ran Gao $\mathbb{D}^{1}$ and Li-Zhen Guo $\mathbb{D}^{2}$ \\ ${ }^{1}$ College of Science, Zhongyuan University of Technology, Zhengzhou 450007, China \\ ${ }^{2}$ School of Mathematics and Statistics, Henan University, Kaifeng 475001, China \\ Correspondence should be addressed to Ran Gao; nygr@163.com
}

Received 17 July 2021; Accepted 15 November 2021; Published 17 December 2021

Academic Editor: Marek Galewski

Copyright (c) 2021 Ran Gao and Li-Zhen Guo. This is an open access article distributed under the Creative Commons Attribution License, which permits unrestricted use, distribution, and reproduction in any medium, provided the original work is properly cited.

\begin{abstract}
The segmentation of weak boundary is still a difficult problem, especially sensitive to noise, which leads to the failure of segmentation. Based on the previous works, by adding the boundary indicator function with $L_{2,1}$ norm, a new convergent variational model is proposed. A novel strategy for the weak boundary image is presented. The existence of the minimizer for our model is given, by using the alternating direction method of multipliers (ADMMs) to solve the model. The experiments show that our new method is robust in segmentation of objects in a range of images with noise, low contrast, and direction.
\end{abstract}

\section{Introduction}

Image segmentation [1-6] is the process of separating objects of interest from each other or backwards to find boundaries of objects. It has become increasingly important in the last decade, due to being a fast-expanding field of applications in image analysis and computer vision. Not only that, it is a fundamental problem in the field of computer vision, because recognition and reconstruction often rely on this information $[7,8]$. There are many circumstances affecting the segmentation results such as the noise, weak edge, and directional texture information, and these effects lead the segmentation problem to be a typical structural ill-posed problem. Many approaches exist to deal with this problem including histogram analysis, region growth, and edge detection $[9,10]$. Up to now, although there are many existing segmentation methods, they still lack the universality. Over the last decade, researchers are constantly exploring new segmentation methods to make image segmentation results as good as possible and variational methods and partial differential equation (PDE) base techniques have been introduced to image segmentation. In this paper, we present a classical method of variation for these problems. Specially, these methods are divided into three methods: threshold-based segmentation [11-13], region-based segmentation [8, 14], and edge-based segmentation $[7,15,16]$. Here, we mainly consider the region-based method and edge-based method, which are uniformed with the most common segmentation method.

The edge-based models such as the GAC model [15-18] mainly utilize the gradient information to direct the contours toward the boundaries of desired objects and then obtain the segmentation image. Therefore, these classes of models are very sensitive to the noise and also show difficulty to detect the weak boundaries. That is to say, when there is strong noise in the image, the boundary between the target and the background must not be obvious enough and the segmentation will fail. In order to overcome this drawback, one way to process a noisy image is by adding a smoothing step prior to segmentation, but doing this also smooths image edges. Moreover, the segmentation result generated by these models is highly dependent on the initial contour placement due to the nonconvexity of the proposed model. For the region-based models $[8,14]$, they mainly incorporate region information so that the image within each region has uniform characteristics such as intensities and textures. Then, these models are more suitable for segmentation of the image with weak boundaries or noise. Furthermore, they are 
also less sensitive to the location of the initial contour. The well-known Mumford-Shah (MS) variational model [7] can achieve both goals simultaneously by using a piecewise smooth representation of an image $[19,20]$ and separate different regions by using a set of close contours (curves in $2 \mathrm{D}$ and surfaces in 3D). However, due to an unknown set of boundaries and its nonconvexity, the numerical experiment of this model has made very hard. With the assumptions of the segmentation regions to be piecewise constant, the Mumford-Shah model can be simplified as the Chan-Vese (CV) model [8, 21, 22]:

$$
\min _{c_{1}, c_{2}, \Gamma} \lambda_{1} \int_{\text {inside }(C)}\left|I_{0}-c_{1}\right|^{2} \mathrm{~d} x+\lambda_{2} \int_{\text {outside }(C)}\left|I_{0}-c_{2}\right|^{2} \mathrm{~d} x+\nu \cdot \text { length }(\Gamma),
$$

where $v, \lambda_{1}$, and $\lambda_{2}$ are positive parameters, $c_{1}$ and $c_{2}$ are the intensity averages of the original image $I_{0}$ inside and outside the contour $\Gamma$, respectively, $\mathrm{d} x$ is the region element, and the term of length is to regularize the contour $C$. Original $\mathrm{CV}$ model (1) only considers to segment two phases as the foreground and the background and then it extends to the multiphase image segmentation problem [23]. Obviously, the CV model can generally get satisfactory results for the images with intensity homogeneity. Simultaneously, the level set method was also proposed to solve problem (1) in [8]. However, due to the nonconvexity of problem (1), we only get the local minima which may lead to wrong levels of detail and scale and then show the sensitivity to the placement of initial contour. Hence, some global minimization active contour models had been proposed to avoid this problem for obtaining the segmentation regions when fixing intensity averages $c_{1}$ and $c_{2}$. Chan et al. [14] proposed a convex relaxation method of the $\mathrm{CV}$ model. Bresson et al. [24] further established theorems to determine the existence of global minimization of the active contour/snake model. Wang et al. [25] proposed a novel global minimization hybrid active contour model. By designing a convex energy functional and the dual algorithm, Xu et al. [26] proposed a global and local active contour model.

Motivated by the Euler-Lagrange equation of problem (1) by introducing the level set function, Chan et al. [14] transformed to consider the following global segmentation model as

$$
\min _{0 \leq u \leq 1} \underbrace{\int_{\Omega}|\nabla u| \mathrm{d} x}_{:=\operatorname{TV}(u)}+\lambda \int_{\Omega}\left(\left(I_{0}-c_{1}\right)^{2}-\left(I_{0}-c_{2}\right)^{2}\right) u \mathrm{~d} x,
$$

where $\lambda$ is an arbitrary positive parameter, $\Omega \subset R^{N}$ is an open set representing the image domain, and TV $(u)$ is the total variation norm of the function $u$. Although the model has the ability of global segmentation, the segmentation for gray-scale inhomogeneous images is invalid. Later, a large number of scholars made a number of improvements to the model, but there are still some problems in the segmentation of image gray-scale unevenness. Recently, Bresson et al. [24] proposed to determine a global minimum of the snake model by enhancing model (2). The enhancement is realized by unifying it with the classic GAC model [27], and then, they considered the following model:

$$
\min _{u \in[0,1]}\{\underbrace{\int_{\Omega} g(x)|\nabla u| \mathrm{d} x}_{:=\left(\mathrm{TV}_{g}\right)(u)}+\lambda \int_{\Omega}\left(\left(I_{0}-c_{1}\right)^{2}-\left(I_{0}-c_{2}\right)^{2}\right) u \mathrm{~d} x\}
$$

where $g(x)$ is the edge indicator function as defined in the above. Obviously, compared with model (2), model (3) segments the image more robustly due to the term $g(x)$. In order to calculate the problem easily, similar to the case in
[28], Bresson et al. [24] transform the above constraint problem into an unconstrained problem at Theorem 3 , and it needs to compute the minimization of the function:

$$
E\left(c_{1}, c_{2}, u\right)=\int_{\Omega} g(x)|\nabla u| \mathrm{d} x+\lambda \int_{\Omega}\left(\left(I_{0}-c_{1}\right)^{2}-\left(I_{0}-c_{2}\right)^{2}\right) u+\alpha \nu(u) \mathrm{d} x
$$

where $\nu(\xi):=\max \{0,2|\xi-(1 / 2)|-1\}$ is an exact penalty function provided that the constant $\alpha$ is chosen large enough compared to $\lambda$ such as $\alpha>(\lambda / 2)\left\|\left(I_{0}-c_{1}\right)^{2}-\left(I_{0}-c_{2}\right)^{2}\right\|_{L^{\infty}(\Omega)}$. Because problem (4) is unconstrained, it includes a nonsmoothing term and a nonlinear term which lead to numerical difficulties by directly employing numerical methods. One efficient method is to introduce a constrained variable $v:=u$ and then get the following form based on the penalty method: 


$$
E\left(c_{1}, c_{2}, u, v\right)=\int_{\Omega} g(x)|\nabla u| \mathrm{d} x+\frac{1}{2 \theta}\|u-v\|_{2}^{2}+\lambda \int_{\Omega}\left(\left(I_{0}-c_{1}\right)^{2}-\left(I_{0}-c_{2}\right)^{2}\right) v+\alpha v(v) \mathrm{d} x
$$

where $\theta>0$ is the penalty parameter. Furthermore, in order to overcome numerical difficulties generated by the $L^{1}$-norm term, an alternative minimization scheme was introduced by adding some auxiliary variables in [24]. However, the convergence analysis of this scheme is lost.
In this section, based on (5), let $\Omega \in R^{n}$ be a bounded open set with Lipschitz boundary and $u$ be a given image. We propose a new model based on Bresson's method [24] as follows:

$$
E\left(c_{1}, c_{2}, u, v\right)=\left\{\int_{\Omega}\|G \nabla u\|_{2,1} \mathrm{~d} x+\frac{1}{2 \theta}\|u-v\|_{2}^{2}+\lambda \int_{\Omega}\left(\left(I_{0}-c_{1}\right)^{2}-\left(I_{0}-c_{2}\right)^{2}\right) v+\alpha \nu(v) \mathrm{d} x\right\}
$$

where $I_{0}, c_{1}$, and $c_{2}$ are defined in (1) and $v(v)$ is defined in (4). Also,

$$
\begin{aligned}
G & =\left(\begin{array}{cc}
g_{x} & 0 \\
0 & g_{y}
\end{array}\right), \\
\|\xi\|_{2,1} & =\sqrt{\left(\xi_{x}\right)^{2}+\left(\xi_{y}\right)^{2}}, \\
\nabla g & =\left(g_{x}, g_{y}\right) .
\end{aligned}
$$

The minimizing energy function $E\left(c_{1}, c_{2}, u, v\right)$ is as follows:

$$
\min _{c_{1}, c_{2}, u, v} E\left(c_{1}, c_{2}, u, v\right)
$$

Our novel contribution is to put an edge indication function into the $L_{2,1}$ norm, and a novel strategy for the image with weak boundaries is present. The existence of the minimizer for (8) is given as follows.

The alterative scheme to solve the minimizer model (8) is as follows:

(1) Choose the original value for $c_{1}^{0}, c_{2}^{0}, u^{0}$, and $v^{0}$, and set $n:=0$.

(2) When the stop criterion is not satisfied, compute the following iteration:

$$
\begin{aligned}
& \left\{\begin{array}{l}
\underset{c_{1}}{\arg \min } \lambda \int_{\Omega}\left(I_{0}-c_{1}\right)^{2} v \mathrm{~d} x \\
\underset{c_{2}}{\arg \min } \lambda \int_{\Omega}-\left(I_{0}-c_{2}\right)^{2} v \mathrm{~d} x
\end{array}\right. \\
& \left\{\begin{array}{l}
\underset{u}{\arg \min }\|G \nabla u\|_{2}^{2}+\frac{1}{2 \theta}\left\|u-v^{n-1}\right\|_{2}^{2}, \\
\underset{v}{\arg \min } \frac{1}{2 \theta}\left\|u^{n}-v\right\|_{2}^{2}+\lambda \int_{\Omega} r_{1}\left(x, c_{1}, c_{2}\right) v+\alpha v(v) \mathrm{d} x .
\end{array}\right.
\end{aligned}
$$

The rest of this paper is organized as follows: In Section 2, the general framework of the proposed method and the concrete solution to each subproblem are presented. In Section 3, numerical algorithm and experimental results to illustrate the effectiveness of our model in image segmentation are given. Finally, we conclude the paper in Section 4.

\section{The Weighted Chan-Vese Model}

In this section, we mainly show the related definitions and properties and solve every subproblem in model (8).

2.1. Basic Notations. Without loss of generality, we present a gray image as an $N \times N$ matrix, that is, $\Omega=\{1,2, \ldots, N\} \times\{1,2, \ldots, N\}$. The Euclidean space $R^{N \times N}$ is denoted as $\mathrm{W}$, let $H: W \times W$, and we define the gradient operator $\nabla: W \longrightarrow H$, for $\forall v \in W$, where $\nabla v$ denotes the gradient operator in the discrete context.

$$
\begin{aligned}
\|u\|_{2,1} & =\sum_{i=1}^{N}\left(\sum_{j=1}^{N}\left(u_{i, j}\right)^{2}\right)^{(1 / 2)}, \\
\|u\|_{2} & =\left(\sum_{i=1}^{N} \sum_{j=1}^{N}\left(u_{i, j}\right)^{2}\right)^{(1 / 2)} .
\end{aligned}
$$

Let $X=R^{N \times N}$ be a finite-dimensional vector space equipped with a standard scalar product for $u, v \in X$ :

$$
\begin{aligned}
& \langle u, v\rangle_{X}=\int_{\Omega} u v \mathrm{~d} x, \\
& \langle v, v\rangle_{X}=\|v\|_{2,1} .
\end{aligned}
$$

For discretization of the gradient operator $\nabla u$, we use standard the finite difference operators with periodic boundary condition as follows:

$$
(\nabla u)_{i, j}=\left(\begin{array}{c}
\left(\nabla_{x}^{+} u\right)_{i, j} \\
\left(\nabla_{y}^{+} u\right)_{i, j}
\end{array}\right)
$$

where 


$$
\begin{aligned}
\left(\nabla_{x}^{+} u\right)_{i, j} & = \begin{cases}u_{i+1, j}-u_{i, j}, & \text { if } 1 \leq i<N, 1 \leq j \leq N, \\
u_{1, j}-u_{i, j}, & \text { if } i=N, 1 \leq j \leq N,\end{cases} \\
\left(\nabla_{y}^{+} u\right)_{i, j} & = \begin{cases}u_{i, j+1}-u_{i, j}, & \text { if } 1 \leq i \leq N, 1 \leq j<N, \\
u_{i, 1}-u_{i, j}, & \text { if } 1 \leq i \leq N, j=N,\end{cases} \\
\left(\nabla_{x}^{-} u\right)_{i, j} & = \begin{cases}u_{i, j}-u_{i-1, j}, & \text { if } 1<i \leq N, 1 \leq j \leq N, \\
u_{i, j}-u_{N, j}, & \text { if } i=1,1 \leq j \leq N,\end{cases} \\
\left(\nabla_{y}^{-} u\right)_{i, j} & = \begin{cases}u_{i, j+1}-u_{i, j}, & \text { if } 1 \leq i \leq N, 1<j \leq N, \\
u_{i, j}-u_{i, N}, & \text { if } 1 \leq i \leq N, j=1 .\end{cases}
\end{aligned}
$$

Furthermore, we define the discrete divergence operator $\operatorname{div} p: H \longrightarrow W$ by using the divergence theorem [27].

$$
\langle\nabla u, p\rangle_{H}=-\langle u, \operatorname{div} p\rangle_{W},
$$

for $\forall u \in R^{N \times N}$, where $p \in R^{N \times N} \times R^{N \times N}$ and div denotes the adjoint operator of $\nabla$; then, we have

$$
\begin{aligned}
& \operatorname{div} p_{i, j}=\left(\nabla_{x}^{-} p\right)_{i, j}+\left(\nabla_{y}^{-} p\right)_{i, j}, \\
& \left|\nabla u_{i, j}\right|=\sqrt{\left(\left(\nabla_{x}^{+}\right)_{i, j}\right)^{2}+\left(\left(\nabla_{y}^{+}\right)_{i, j}\right)^{2} .}
\end{aligned}
$$

2.2. The Minimization Problem. In this section, we first review some definitions and facts which will be used in our convergence analysis Algorithm 1. These related contents can be found in the books $[29,30]$. We use ADMM to solve every subproblem.

2.2.1. Energy Minimization with respect to $c_{1}$ and $c_{2}$. We fixed $u$ and $v$ in order to determine an exact solution of $c_{1}$ and $c_{2}$; then, the minimization of $E\left(c_{1}, c_{2}, u, v\right)$ with respect to $c_{1}$ and $c_{2}$ is as follows:

$$
\begin{aligned}
& \underset{c_{1}}{\arg \min } \lambda \int_{\Omega}\left(I_{0}-c_{1}\right)^{2} v \mathrm{~d} x, \\
& \underset{c_{2}}{\arg \min } \lambda \int_{\Omega}-\left(I_{0}-c_{2}\right)^{2} v \mathrm{~d} x .
\end{aligned}
$$

Then, we can obtain

$$
c_{1}=c_{2}=\frac{\int I_{0}}{\int I} .
$$

2.2.2. Energy Minimization with respect to $u$ and $v$. For any given $c_{1}$ and $c_{2}$, we have the problem

$$
\underset{u, v}{\arg \min }:=\left\{\int_{\Omega}\|G \nabla u\|_{2,1} \mathrm{~d} x+\frac{1}{2 \theta}\|u-v\|_{2}^{2}+\lambda \int_{\Omega}\left(\left(I_{0}-c_{1}\right)^{2}-\left(I_{0}-c_{2}\right)^{2}\right) v+\alpha \nu(v) \mathrm{d} x\right\} .
$$

Problem (18) is convex and is not strictly convex about $u$ and $v$, so its local minimizer is also the global minimizer. One of the effective methods is to change it into an unconstrained minimization problem according to the theorems in $[14,24]$.
Obviously, it is easy to find that the objective function in problem (18) is strictly convex and coercive, so it owns a unique solution $\left(u^{*}, v^{*}\right)$. Formally, problem (18) including two variables $u$ and $v$ as a coupled problem, and the classical method is to divide it into two subproblems. Thus, we alteratively consider the following minimization problem:

$$
\begin{aligned}
S(v):=u & =\min _{u}\|G \nabla u\|_{2}^{2}+\frac{1}{2 \theta}\|u-v\|_{2}^{2}, \\
\mathscr{T}(u) & :=v=\min _{v} \frac{1}{2 \theta}\|u-v\|_{2}^{2}+\lambda \int_{\Omega}\left(\left(I_{0}-c_{1}\right)^{2}-\left(I_{0}-c_{2}\right)^{2}\right) v+\alpha v(v) \mathrm{d} x .
\end{aligned}
$$

Theorem 1. The following assertions hold:

$$
u=v-\mathscr{P}_{\theta \mathcal{S}}(v)
$$

(1) The solution of problem (19) is given by

where $\mathscr{P}$ is the project operator and $\mathcal{S}$ is defined by

$$
\mathcal{S}=\operatorname{closure}\left\{\operatorname{div} \xi(x): \xi(x) \in C_{c}^{1}\left(\Omega, R^{2}\right),|\xi(x)| \leq g(x), \forall x \in \Omega\right\}
$$

(2) The solution of problem (17) satisfies

$$
v=\min \left\{\max \left\{u-\theta \lambda r_{1}\left(x, c_{1}, c_{2}\right), 0\right\}, 1\right\},
$$


(1)Choose the original value $u^{0}$ and $v^{0}$, and set $n:=0$.

(2) While the stop criterion is not satisfied, compute the following iteration: $\left\{\begin{array}{l}\operatorname{argmin}_{u}\|G \nabla u\|_{2}^{2}+(1 / 2 \theta)\left\|u-v^{n-1}\right\|_{2}^{2} \\ \operatorname{argmin}_{v}(1 / 2 \theta)\left\|u^{n}-v\right\|_{2}^{2}+\lambda \int_{\Omega} r_{1}\left(x, c_{1}, c_{2}\right) v+\alpha v(v) \mathrm{d} x\end{array}\right.$

Algorithm 1: The alterative scheme to solve the minimizer models (19) and (20).

$$
\text { where } r_{1}\left(x, c_{1}, c_{2}\right)=\left(I_{0}-c_{1}\right)^{2}-\left(I_{0}-c_{2}\right)^{2} \text {. }
$$

Remark 1. Obviously, problem (19) is a generated ROF model by adding a weighted function $g(x)$ to the regularization term and so we can get the solution based on the scheme in $[27,31,32]$. For problem (20), it is similar to what happens in [28] for this circumstance; we notice that it is equivalent to the following minimization problem:

$$
\min _{v} \frac{1}{2 \theta}\|u-v\|_{2}^{2}+\lambda \int_{\Omega} r_{1}\left(x, c_{1}, c_{2}\right) u+\delta_{\mathscr{C}}(v) \mathrm{d} x,
$$

where $\delta$ is the indicator function defined by

$$
\delta(x)= \begin{cases}0, & \text { if } x \in \mathscr{C}, \\ +\infty, & \text { if } x \notin \mathscr{C},\end{cases}
$$

with $\mathscr{C}=[0,1]$. Thus, we can easily find that the solution of problem (20) satisfies condition (23).

Definition 1. The proximal operator of a proper, convex, semicontinuous function $\varphi$ on $R^{n}$ is defined for $x \in R^{n}$ by

$$
\operatorname{prox}_{\gamma \varphi \circ B} x:=\underset{y \in R^{n}}{\arg \min }\left\{\frac{1}{2 \gamma}\|y-x\|_{2}^{2}+\varphi(B y)\right\},
$$

where $B: R^{n} \longrightarrow R^{n}$ is a bounded linear operator. Furthermore, when $B=I$, it is the classical proximal operator defined in [33].

Definition 2. Let $\varphi$ be a real-value convex function on $R^{n}$. The subdifferential of $\varphi$ at $x \in R^{n}$ is defined by

$$
\partial \varphi(x):=\left\{y: y \in R^{n} \text { and } \varphi(z) \geq \varphi(x)+(y, z-x) \forall z \in R^{n}\right\} .
$$

The elements in $\partial \varphi(x)$ are called subgradients. Especially, $\partial \varphi(x)=\nabla \varphi(x)$ when $\varphi(x)$ is differential.

Definition 3. An operator $Q$ : $R^{n} \longrightarrow R^{n}$ is firmly nonexpansive if it satisfies one of the following equivalent conditions:

(i) $\|\mathbb{Q} x-\mathbb{Q} y\|_{2}^{2} \leq(\mathbb{Q} x-\mathbb{Q} y, x-y), \forall(x, y) \in R^{n} \times R^{n}$

(ii) $\|\mathbb{Q} x-\mathbb{Q} y\|_{2}^{2} \leq\|x-y\|_{2}^{2}-\|(I-Q) x-(I-Q) y\|_{2}^{2}$, $\forall(x, y) \in R^{n} \times R^{n}$

Definition 4. An operator $Q: R^{n} \longrightarrow R^{n}$ is nonexpansive if for any $(x, y) \in R^{n} \times R^{n}$, we have

$$
\|Q \mathcal{Q} x-Q\|_{2} \leq\|x-y\|_{2} \text {. }
$$

It follows that a firmly nonexpansive operator $\mathscr{P}$ is nonexpansive. Based on the proof of the proximal operator in [34], we obtain the similar result of the proximal operator defined in Definition 1.

Lemma 1. The proximal operator prox ${ }_{\gamma \varphi B}$ defined in Definition 1 is firmly nonexpansive.

Proof. Following the definition of the proximity in Definition 1 and the chain rule, we can get

$$
\left\{\begin{array}{l}
\varphi\left(B \circ \operatorname{prox}_{\gamma \varphi \circ B} y\right)-\varphi\left(B \circ \operatorname{prox}_{\gamma \varphi \circ B} x\right) \geq \frac{1}{\gamma}\left(x-\operatorname{prox}_{\gamma \varphi \circ B} x, \operatorname{prox}_{\gamma \varphi \circ B} y-\operatorname{prox}_{\gamma \varphi \circ B} x\right), \\
\varphi\left(B \circ \operatorname{prox}_{\gamma \varphi \circ B} x\right)-\varphi\left(B \circ \operatorname{prox}_{\gamma \varphi \circ B} y\right) \geq \frac{1}{\gamma}\left(y-\operatorname{prox}_{\gamma \varphi \circ B} y, \operatorname{prox}_{\gamma \varphi \circ B} x-\operatorname{prox}_{\gamma \varphi \circ B} y\right) .
\end{array}\right.
$$

We obtain

$$
\left\|\operatorname{prox}_{\gamma \varphi \circ B} x-\operatorname{prox}_{\gamma \varphi \circ B} y\right\|_{2}^{2} \leq\left(\operatorname{prox}_{\gamma \varphi \circ B} x-\operatorname{prox}_{\gamma \varphi \circ B} y, x-y\right),
$$

which implies the nonexpansion of the operator $\operatorname{prox}_{\gamma \varphi \circ B}$.
Corollary 1. The operator $\mathcal{S}$ defined in minimization problem (19) is nonexpansive.

Proof. Based on above definitions, set $B=g(x)|\nabla u|$; then, the operator $\mathscr{T}$ is the proximal operator of $\int_{\Omega} g(x)|\nabla u| \mathrm{d} x$. Furthermore, we can get $\mathcal{S}=$ pro $_{|\cdot|_{L}{ }^{\circ} B}$. According to Lemma 1 , the operator $\mathcal{S}$ is nonexpansive. 
Lemma 2. Assume that $\mathscr{C} \subset R^{n}$ is a closed convex set and $\mathscr{P}_{\mathscr{C}}(\cdot): R^{n} \longrightarrow \mathscr{C}$ is a projection operator; then, the projector $\mathscr{P}_{\mathscr{C}}(\cdot)$ is firmly nonexpansive.

Proof. Let $x \in R^{n}$ and $\forall y \in \mathscr{C}$; we have

$$
\left(y-\mathscr{P}_{\mathscr{C}}(x), x-\mathscr{P}_{\mathscr{C}}(x)\right) \leq 0 .
$$

Since $\mathscr{P}_{\mathscr{C}}(y)=y$ while $y \in \mathscr{C}$, we can get

$$
\left(\mathscr{P}_{\mathscr{C}}(y)-\mathscr{P}_{\mathscr{C}}(x), x-y+\mathscr{P}_{\mathscr{C}}(y)-\mathscr{P}_{\mathscr{C}}(x)\right) \leq 0 \text {. }
$$

This yields

$$
\left\|\mathscr{P}_{\mathscr{C}}(x)-\mathscr{P}_{\mathscr{C}}(y)\right\|_{2}^{2} \leq\left(x-y, \mathscr{P}_{\mathscr{C}}(x)-\mathscr{P}_{\mathscr{C}}(y)\right) \leq 0 .
$$

Corollary 2. The operator $\mathscr{T}$ defined in minimization problem (20) is firmly nonexpansive.

Proof. Obviously, from Theorem 1 and Remark 1, we derive that

$$
\mathscr{T}(u):=v=\mathscr{P}_{[0,1]}\left(u-\theta \lambda r_{1}\left(x, c_{1}, c_{2}\right)\right) .
$$

It follows from Lemma 2 that $\mathscr{T}$ is firmly nonexpansive.

In view of the above results, we know that $\mathscr{R}_{1}(\cdot)=\mathcal{S}(\mathscr{T}(\cdot))$ and $\mathscr{R}_{2}(\cdot)=\mathscr{T}(\mathcal{S}(\cdot))$ are firmly nonexpansive.

Lemma 3. Let $u^{(k)}$ and $v^{(k)}$ be generated by (2) and (3), respectively. Then, $\sum_{k=0}^{\infty}\left\|u^{(k)}-u^{(k+1)}\right\|_{L^{2}}^{2}$ and $\sum_{k=0}^{\infty} \| v^{(k)}-$ $v^{(k+1)} \|_{L^{2}}^{2}$ are convergent.

Proof. Denote $E_{1}(u, v)=\|u-v\|_{L^{2}}^{2}$ and $E_{2}(v)=\left\|\nabla_{x} v\right\|_{L^{1}}+$ $\left\|\nabla_{y} v\right\|_{L^{1}}$. We have

$$
E(u, v)=\int_{\Omega}\left(u+g e^{-u}\right) \mathrm{d} x \mathrm{~d} y+\beta_{1} E_{1}(u, v)+\beta_{2} E_{2}(v) .
$$

Hence,

$$
E\left(u^{(k+1)}, v^{(k)}\right)-E\left(u^{(k+1)}, v^{(k+1)}\right)=\beta_{1}\left(E_{1}\left(u^{(k+1)}, v^{(k)}\right)-E_{1}\left(u^{(k+1)}, v^{(k+1)}\right)\right)+\beta_{2}\left(E_{2}\left(v^{(k)}\right)-E_{2}\left(v^{(k+1)}\right)\right) .
$$

Consider the Taylor series expansion of $E_{1}(u, v)$ in the second variable, i.e.,

$$
\begin{aligned}
E_{1}\left(u^{(k+1)}, v^{(k)}\right)= & E_{1}\left(u^{(k+1)}, v^{(k+1)}\right)+\left(v^{(k)}-v^{(k+1)}\right)^{T} \frac{\partial E_{1}}{\partial v}\left(u^{(k+1)}, v^{(k+1)}\right) \\
& +\frac{1}{2}\left(v^{(k)}-v^{(k+1)}\right) \frac{\partial^{2} E_{1}}{\partial v^{2}}\left(u^{(k+1)}, v^{(k+1)}\right)\left(v^{(k)}-v^{(k+1)}\right) . \\
& E_{2}\left(v^{(k)}\right) \geq E_{2}\left(v^{(k+1)}\right)+\left(v^{(k)}-v^{(k+1)}\right)^{T} \frac{\partial E_{2}}{\partial v}\left(v^{(k+1)}\right) .
\end{aligned}
$$

Here, $x^{T}$ denotes the transpose of $x$. We notice that $E_{1}$ is quadratic about $v$. Then, $\left(\partial^{2} E_{1} / \partial v^{2}\right) \equiv 2 I$ where $I$ is the identity matrix. Moreover, since $E_{2}$ is a convex function, we

Combining (36), (37), and (38), we obtain get

$$
\begin{aligned}
& \begin{aligned}
E\left(u^{(k+1)}, v^{(k)}\right)-E\left(u^{(k+1)}, v^{(k+1)}\right) \geq\left(u^{(k)}-u^{(k+1)}\right)^{T}\left(\beta_{1} \frac{\partial E_{1}}{\partial v}\left(u^{(k+1)}, v^{(k+1)}\right)\right. \\
\left.+\beta_{2} \frac{\partial E_{2}}{\partial v}\left(v^{(k+1)}\right)\right)+\beta_{1}\left\|v^{(k)}-v^{(k+1)}\right\|_{L^{2}}^{2} .
\end{aligned} \\
& \qquad \frac{\partial E}{\partial v}=\beta_{1} \frac{\partial E_{1}}{\partial v}+\beta_{2} \frac{\partial E_{2}}{\partial v} .
\end{aligned}
$$

The subdifferential of $E$ with respect to $v$ is equivalent to the vector sum of the subdifferential of $E_{1}$ and $E_{2}$ about $v$, i.e.,
Since $v^{(k+1)}$ is the minimizer of $E\left(u^{(k+1)}, v\right)$, we have 


$$
\frac{\partial E}{\partial v}\left(u^{(k+1)}, v^{(k+1)}\right)=0
$$

that is

$$
\beta_{1} \frac{\partial E_{1}}{\partial v}\left(u^{(k+1)}, v^{(k+1)}\right)+\beta_{2} \frac{\partial E_{2}}{\partial v}\left(z^{(k+1)}, v^{(k+1)}\right)=0
$$

Therefore, the first term in the right hand side of (39) is zero. When we solve successive minimization problems (19) and (20), we note that $E\left(u^{(k)}, v^{(k)}\right) \geq E\left(u^{(k+1)}, v^{(k)}\right)$. Hence, we get

$$
E\left(u^{(k)}, v^{(k)}\right)-E\left(u^{(k+1)}, v^{(k+1)}\right) \geq E\left(u^{(k+1)}, v^{(k)}\right)-E\left(u^{(k+1)}, v^{(k+1)}\right) \geq \beta_{1}\left\|v^{(k)}-v^{(k+1)}\right\|_{L^{2}}^{2}
$$

It follows that the partial sum of the sequence $\left\{\left\|v^{(k)}-v^{(k+1)}\right\|_{L^{2}}^{2}\right\}_{k=0}^{\infty}$ is bounded. Thus, the infinite series $\sum_{k=0}^{\infty}\left\|v^{(k)}-v^{(k+1)}\right\|_{L^{2}}^{2}$ is convergent.

Let $E_{3}(u)=\int_{\Omega}\left(u+g e^{-u}\right) \mathrm{d} x \mathrm{~d} y$. By considering $E\left(u^{(k)}, v^{(k)}\right)-E\left(u^{(k+1)}, v^{(k)}\right)$ and using the similar method, we can prove that $\sum_{k=0}^{\infty}\left\|u^{(k)}-u^{(k+1)}\right\|_{L^{2}}^{2}$ is convergent.

Definition 5. A operator $\mathscr{P}: R^{n} \longrightarrow R^{n}$ is asymptotically regular if for any $x$ in $R^{n}$, the sequence $\left\{\mathscr{P}^{k+1} x-\mathscr{P}^{k} x\right\}$ tends to zero as $k \longrightarrow \infty$.

Based on Lemma 3, we get the following result.

Lemma 4. For any initial values $v^{(0)}$ and $u^{(0)}$, assume $u^{(k)}$ and $v^{(k)}$ are generated by (19) and (20), respectively; then, $\mathscr{T}_{1}$ and $\mathscr{T}_{2}$ are asymptotically regular.

Proof. According to Lemma 3, we obtain

$$
\begin{aligned}
& \lim _{k \longrightarrow \infty}\left\|v^{(k)}-v^{(k+1)}\right\|_{L^{2}}^{2}=0, \\
& \lim _{k \longrightarrow \infty}\left\|u^{(k)}-u^{(k+1)}\right\|_{L^{2}}^{2}=0 .
\end{aligned}
$$

As $v^{(k)}=\mathscr{T}_{1}\left(v^{(k-1)}\right)$ and $u^{(k)}=\mathscr{T}_{2}\left(u^{(k-1)}\right)$, by using the recurrence method, we have

$$
\begin{aligned}
& v^{(k)}=\mathscr{T}_{1}^{k}\left(v^{(0)}\right), \\
& u^{(k)}=\mathscr{T}_{2}^{k}\left(u^{(0)}\right) .
\end{aligned}
$$

Therefore, we get

$$
\begin{aligned}
& \lim _{k \longrightarrow \infty}\left\|\mathscr{T}_{1}^{k+1}\left(v^{(0)}\right)-\mathscr{T}_{1}^{k}\left(v^{(0)}\right)\right\|_{L^{2}}=0, \\
& \lim _{k \longrightarrow \infty}\left\|\mathscr{T}_{2}^{k+1}\left(u^{(0)}\right)-\mathscr{T}_{2}^{k}\left(u^{(0)}\right)\right\|_{L^{2}}=0 .
\end{aligned}
$$

This indicates that $\mathscr{T}_{1}$ and $\mathscr{T}_{2}$ are asymptotically regular.

Lemma 5. Suppose the unique minimizer of $E(u, v)$ is $\left(u^{*}, v^{*}\right)$. Then, $u^{*}$ and $v^{*}$ are the unique fixed points of $\mathscr{T}_{2}$ and $\mathscr{T}_{1}$, respectively.
Proof. Since $E(u, v)$ is differentiable with respect to $u$ and $v$ separately, we obtain

$$
\left(\begin{array}{l}
\frac{\partial E}{\partial u}\left(u^{*}, v^{*}\right) \\
\frac{\partial E}{\partial v}\left(u^{*}, v^{*}\right)
\end{array}\right)=\left(\begin{array}{l}
0 \\
0
\end{array}\right)
$$

This implies that

$$
\begin{aligned}
& u^{*}=\mathscr{R}\left(v^{*}\right)=\underset{u}{\arg \min } E\left(u, v^{*}\right), \\
& v^{*}=\mathcal{S}\left(u^{*}\right)=\underset{v}{\arg \min } E\left(u^{*}, v\right) .
\end{aligned}
$$

We easily get $u^{*}=\mathscr{R}\left(v^{*}\right)=\mathscr{T}_{2}\left(u^{*}\right)$ and $v^{*}=\mathcal{S}\left(u^{*}\right)=\mathscr{T}_{1}\left(v^{*}\right)$. Therefore, $u^{*}$ and $v^{*}$ are the corresponding fixed points of $\mathscr{T}_{1}$ and $\mathscr{T}_{2}$.

On the other hand, we note that $E(u, v)$ is strictly convex and differentiable about $u$ and $v$, respectively. Therefore, the fixed points of $\mathscr{T}_{1}$ and $\mathscr{T}_{2}$ are the minimizers of $E(u, v)$. By virtue of the uniqueness of the minimizer of $E(u, v), \mathscr{T}_{1}$ and $\mathscr{T}_{2}$ show a unique fixed point separately. That is to say, that $u^{*}$ and $v^{*}$ are the unique fixed points of $\mathscr{T}_{2}$ and $\mathscr{T}_{1}$, respectively. result.

According to Theorem 1 in [7], we get the following

Theorem 2. The sequence $\left\{\left(u^{n}, v^{n}\right)\right\}$ generated by Algorithm 1 converges to the solution $\left(u^{*}, v^{*}\right)$ of problems (19) and (20).

Proof. We should first notice the fact that

$$
\begin{aligned}
v^{n} & =\min _{v} F\left(u^{n-1}, v\right) \\
u^{n} & =\min _{u} F\left(u, v^{n}\right),
\end{aligned}
$$

so we can get

$$
F\left(u^{n}, v^{n}\right) \leq F\left(u^{n-1}, v^{n}\right) \leq F\left(u^{n-1}, v^{n-1}\right) .
$$

In particular, the sequence $F\left(u^{n}, v^{n}\right)$ is nonincreasing. 
Theorem 3. For any initial values $v^{(0)}$ and $u^{(0)}$, assume $u^{(k)}$ and $v^{(k)}$ are generated by (2) and (3), respectively; then $u^{(k)}$ and $v^{(k)}$ converge to the corresponding fixed points of $\mathscr{T}_{2}$ and $\mathscr{T}_{1}$, i.e., $\left(u^{(k)}, v^{(k)}\right)$ converges to $\left(u^{*}, v^{*}\right)$ which is the unique minimizer of $E(u, v)$, as $k \longrightarrow \infty$.

Proof. In view of Lemmas 1-5, we know that $\mathscr{T}_{1}: R^{n} \longrightarrow R^{n}$ and $\mathscr{T}_{2}: R^{n} \longrightarrow R^{n}$ are nonexpansive asymptotically regular mappings and have fixed points. By using Theorem 1 in [7], we get that $u^{(k)}$ and $v^{(k)}$ converge, respectively, to the fixed points of $\mathscr{T}_{2}$ and $\mathscr{T}_{1}$, i.e., $\left(u^{(k)}, v^{(k)}\right)$ converges to $\left(u^{*}, v^{*}\right)$ which is the unique minimizer of $E(u, v)$, as $k \longrightarrow \infty$.

\section{General Framework of the Proposed Model}

Now, we discuss the solution to each variable separately; without loss of generality, we use the ADMM method to solve minimization problem (18).

$$
\begin{aligned}
& u:=\min _{u} \int_{\Omega}\|G \nabla u\|_{2,1} \mathrm{~d} x+\frac{1}{2 \theta}\|u-v\|_{2}^{2}, \\
& v:=\min _{v} \frac{1}{2 \theta}\|u-v\|_{2}^{2}+\lambda \int_{\Omega}\left(\left(I_{0}-c_{1}\right)^{2}-\left(I_{0}-c_{2}\right)^{2}\right) v+\alpha v(v) \mathrm{d} x .
\end{aligned}
$$

Then, in the following sections, we obtain the solutions to problems (51) and (52).

3.1. Algorithm for Solving (51). In this section, we solve model (51) about $u$; because the model is not easy to solve, we use ADMM to solve problem (51). We can take $\mathbf{w}=\nabla u$ and $\mathbf{T}=G \mathbf{w}$ in our model, and by introducing two variables, we can obtain the following constrained problem:

$$
\left\{\begin{array}{l}
\min _{u, \mathbf{w}, \mathbf{T}} \int_{\Omega}\|\mathbf{T}\|_{2,1} \mathrm{~d} x+\frac{1}{2 \theta}\|u-v\|_{L_{2}}^{2} \\
\text { s.t. } \quad \mathbf{w}=\nabla u, \mathbf{T}=G \mathbf{w},
\end{array}\right.
$$

where

$$
\begin{aligned}
& \mathbf{w}:=\left(\begin{array}{l}
\mathbf{w}_{x} \\
\mathbf{w}_{y}
\end{array}\right)=\left(\begin{array}{l}
u_{x} \\
u_{y}
\end{array}\right), \\
& \mathbf{T}:=\left(\begin{array}{c}
\mathbf{T}_{x} \\
\mathbf{T}_{y}
\end{array}\right)=\left(\begin{array}{cc}
g_{x} & 0 \\
0 & g_{y}
\end{array}\right)\left(\begin{array}{c}
\mathbf{w}_{x} \\
\mathbf{w}_{y}
\end{array}\right) .
\end{aligned}
$$

By using the augmented Lagrangian method to turn the constrained problem into the unconstrained problem, we have the augmented Lagrangian function as follows:

$$
\begin{aligned}
\mathscr{L}\left(u, \mathbf{w}, \mathbf{T} ; \beta_{1}, \beta_{2}\right)= & \int_{\Omega}\|\mathbf{T}\|_{2,1} \mathrm{~d} x+\frac{1}{2 \theta}\|u-v\|_{L_{2}}^{2}+\left\langle\beta_{1}, \mathbf{w}-\nabla u\right\rangle+\frac{\gamma_{1}}{2}\|\mathbf{w}-\nabla u\|_{L_{2}}^{2} \\
& +\left\langle\beta_{2}, \mathbf{T}-G \mathbf{w}\right\rangle+\frac{\gamma_{2}}{2}\|\mathbf{T}-G \mathbf{w}\|_{L_{2}}^{2}
\end{aligned}
$$

where $\beta_{1}$ and $\beta_{2}$ are Lagrangian multiplier that can alternatively be regarded as the dual variables of problem $\mathbf{w}$ and $\mathbf{T}$ and $\gamma_{1}>0$ and $\gamma_{2}>0$ are penalty parameters which are constant that should be chosen properly. We convert it into the maximum minimization problem and simplify

$$
\min _{u, \mathbf{w}, \mathbf{T}} \max _{\beta_{1}, \beta_{2}} \int_{\Omega}\|\mathbf{T}\|_{2,1} \mathrm{~d} x+\frac{1}{2 \theta}\|u-v\|_{L_{2}}^{2}+\frac{\gamma_{1}}{2}\left\|\mathbf{w}-\nabla u+\frac{\beta_{1}}{\gamma_{1}}\right\|_{L_{2}}^{2}+\frac{\gamma_{2}}{2}\left\|\mathbf{T}-G \mathbf{w}+\frac{\beta_{2}}{\gamma_{2}}\right\|_{L_{2}}^{2} .
$$




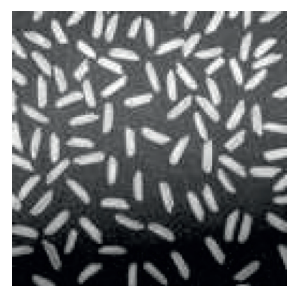

(a)

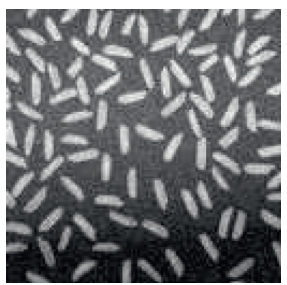

(e)

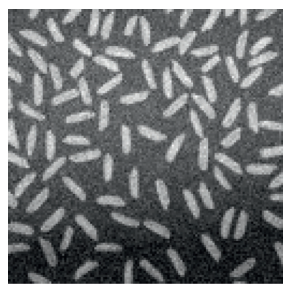

(i)

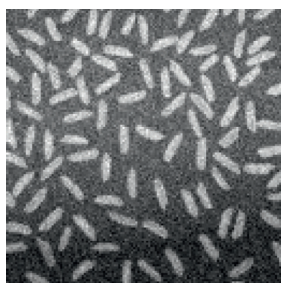

(m)

Original image

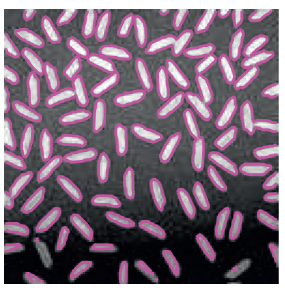

(b)

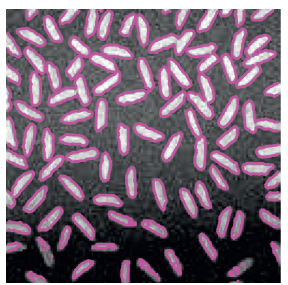

(f)

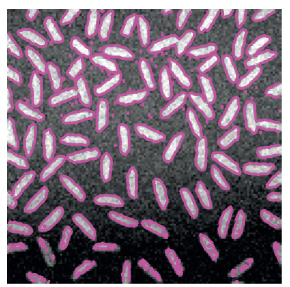

(j)

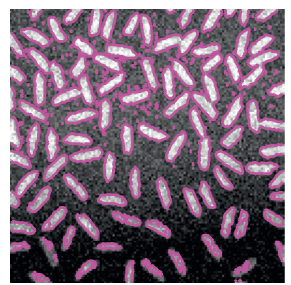

(n)

Chan - Vese method

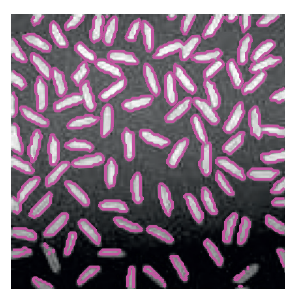

(c)

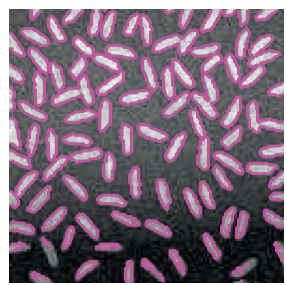

(g)

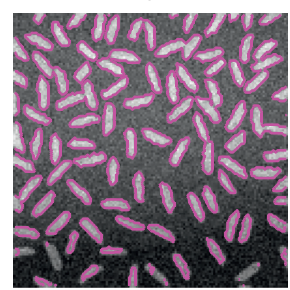

(k)

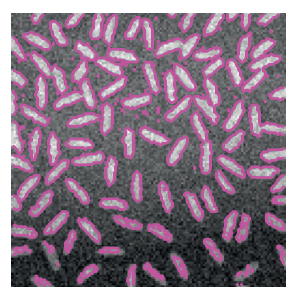

(o)

Bresson method

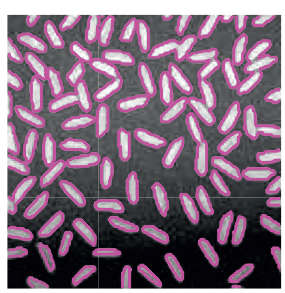

(d)

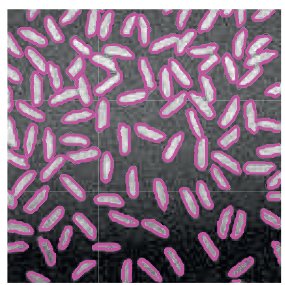

(h)

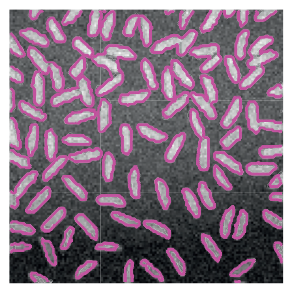

(1)

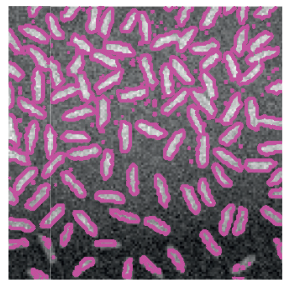

(p)

Our method

FIGURE 1: Illustration of without noisy image and three noisy image and the performance of all the three models by 3000 iterations. First row: the result of three methods for the original image segmentation without noise. Second row: the result of three methods for the original image segmentation with 0.1 noise. Third row: the result of three methods for the original image segmentation with 0.15 noise. Fourth row: the result of three methods for the original image segmentation with 0.2 noise.

In the following, we use ADMM to solve each subproblem about (56). First, the minimum energy function $E(u, \mathbf{w}, \mathbf{T})$ is as follows:

$$
\min _{u, \mathbf{w}, \mathbf{T}} E(u, \mathbf{w}, \mathbf{T}) .
$$

The maximum energy function $E\left(\beta_{1}, \beta_{2}\right)$ is as follows:

$$
\max _{\beta_{1}, \beta_{2}} E\left(\beta_{1}, \beta_{2}\right) \text {. }
$$

Then, we solve minimization and maximization problems by using ADMM.

(1) Subminimization with respect to. T. We fixed $u$ and $\mathbf{w}$ in order to determine an exact solution of $\mathbf{T}$; then, we have the minimization of $\mathbf{T}$ with respect to $u$ and $\mathbf{w}$ as follows:

$$
\lim _{\mathbf{T}} \int_{\Omega}\|\mathbf{T}\|_{21} \mathrm{~d} x+\frac{\gamma_{2}}{2}\left\|\mathbf{T}-G \mathbf{w}+\frac{\beta_{2}}{\gamma_{2}}\right\|_{L_{2}}^{2} .
$$

In order to solve $\mathbf{T}$, we use the soft threshing operator defined in [35] and obtain

$$
\mathbf{T}=\max \left\{\left\|G \mathbf{w}-\frac{\beta_{2}}{\gamma_{2}}\right\|_{1}-\frac{1}{\gamma_{2}}, 0\right\} \frac{G \mathbf{w}-\left(\beta_{2} / \gamma_{2}\right)}{\left\|G \mathbf{w}-\left(\beta_{2} / \gamma_{2}\right)\right\|_{1}} .
$$

For the corresponding Lagrangian multiplier $\beta_{2}$, we update it by using the gradient ascent method:

$$
\beta_{2}=\beta_{2}+\gamma_{2}(\mathbf{T}-G \mathbf{w}) \text {. }
$$

(2) Subminimization with respect to $\mathbf{w}$. We fixed $u$ and $\mathbf{T}$ in order to determine an exact solution of $\mathbf{w}$; the minimization of $\mathbf{T}$ with respect to $u$ and $\mathbf{w}$ is as follows:

$$
\min _{\mathbf{w}} \frac{\gamma_{1}}{2}\left\|\mathbf{w}-\nabla u+\frac{\beta_{1}}{\gamma_{1}}\right\|_{L_{2}}^{2}+\frac{\gamma_{2}}{2}\left\|\mathbf{T}-G \mathbf{w}+\frac{\beta_{2}}{\gamma_{2}}\right\|_{L_{2}}^{2},
$$

and then we calculate the equation about the $\mathbf{w}$ and obtain 


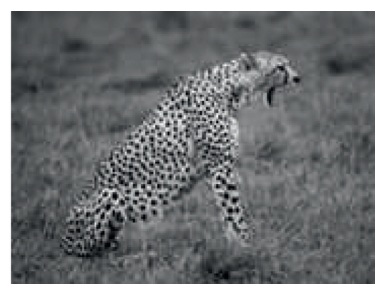

(a)

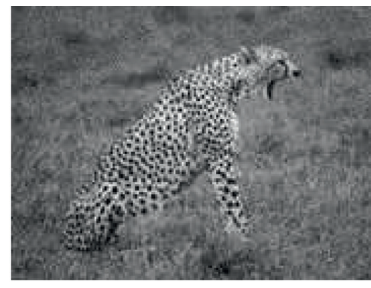

(e)

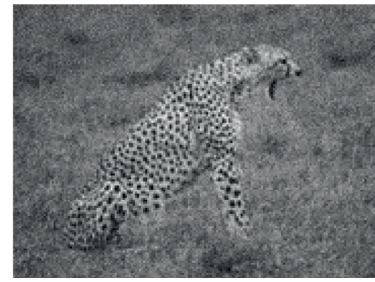

(i)

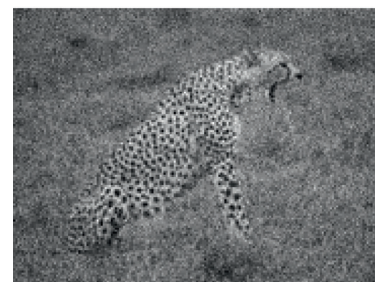

(m)

Original image

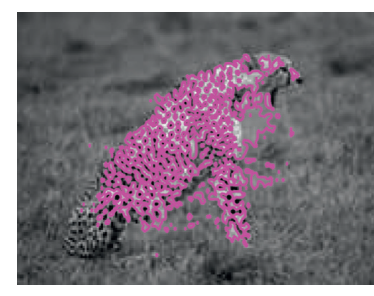

(b)

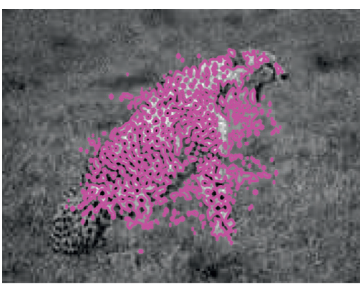

(f)

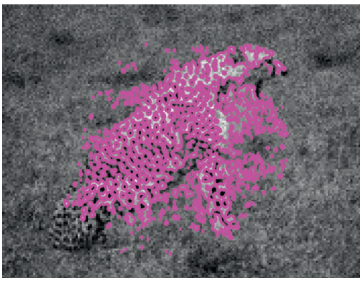

(j)

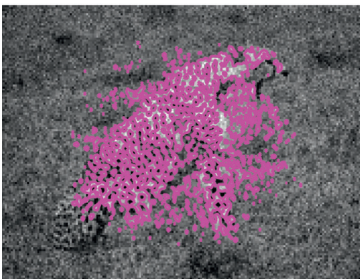

(n)

Chan - Vese method

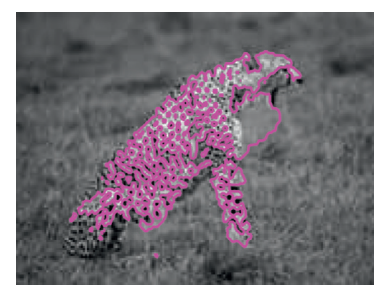

(c)

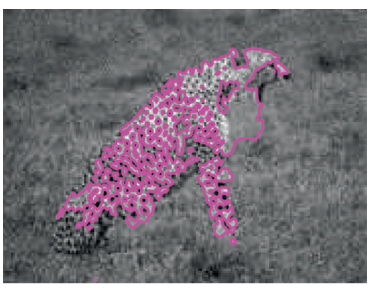

(g)

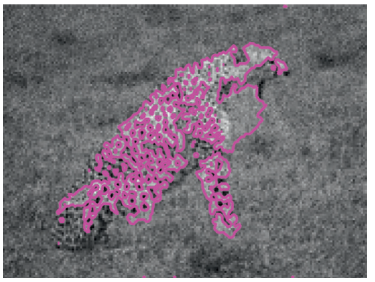

(k)

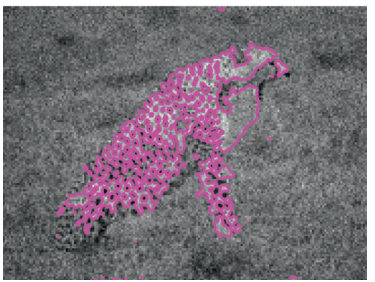

(o)

Bresson method

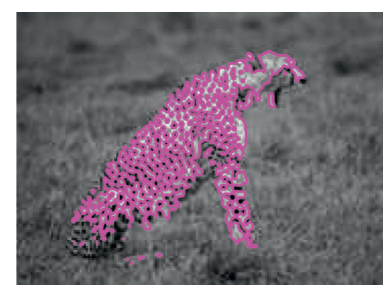

(d)

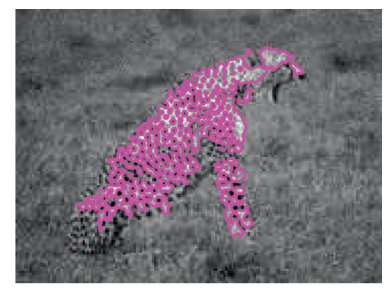

(h)

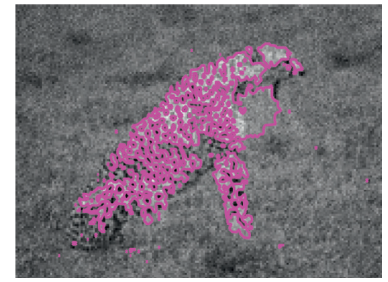

(l)

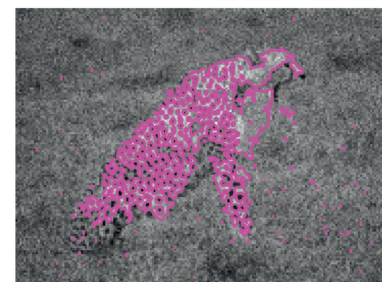

$(\mathrm{p})$

Our method

FIGURE 2: Illustration of without noisy natural image and three noisy image and the performance of all the three models by 3000 iterations. First row: the result of three methods for the original image segmentation without noise. Second row: the result of three methods for the original image segmentation with 0.1 noise. Third row: the result of three methods for the original image segmentation with 0.15 noise. Fourth row: the result of three methods for the original image segmentation with 0.2 noise.

$$
\gamma_{1}\left(\mathbf{w}-\nabla u+\frac{\beta_{1}}{\gamma_{1}}\right)-\gamma_{2} G^{T}\left(\mathbf{T}-G \mathbf{w}+\frac{\beta_{2}}{\gamma_{2}}\right)=0 .
$$

Simplifying equation (63), we can obtain

$$
\mathbf{w}=\frac{\gamma_{2} G\left(T+\left(\beta_{2} / \gamma_{2}\right)\right)+\gamma_{1}\left(\nabla u-\left(\beta_{1} / \gamma_{1}\right)\right)}{\gamma_{1}+\gamma_{2} G^{2}} \text {. }
$$

For the corresponding Lagrangian multiplier $\beta_{1}$, we update it by using the gradient ascent method:

$$
\beta_{1}=\beta_{1}+\gamma_{1}(\mathbf{w}-\nabla \mathbf{u}) \text {. }
$$

(3) Subminimization with respect to $u$. We fixed $\mathbf{T}$ and $\mathbf{w}$ in order to determine an exact solution of $u$; we begin by considering the minimization of (55) with respect to $u$, and then,

$$
\min _{u} \frac{1}{2 \theta}\|u-v\|_{L_{2}}^{2}+\frac{\gamma_{1}}{2}\left\|\mathbf{w}-\nabla u+\frac{\beta_{1}}{\gamma_{1}}\right\|_{L^{2}}^{2} .
$$

Then, using the divergence theorem in the equation about $u$, we obtain

$$
\frac{1}{\theta}(u-v)+\gamma_{1} \operatorname{div}\left(\mathbf{w}-\nabla u+\frac{\beta_{1}}{\gamma_{1}}\right)=0
$$

Similarly, due to periodic boundary condition being imposed, we can solve $u$ efficiently by the fast Fourier transform (FFT). This yields the following solution:

$$
u=\mathscr{F}^{-1}\left(\frac{\mathscr{F}(v)-\theta \gamma_{1} \mathscr{F}(\operatorname{div})\left(\mathbf{w}+\left(\beta_{1} / \gamma_{1}\right)\right)}{1-\theta \gamma_{1} \mathscr{F}(\Delta)}\right) .
$$

with $\mathscr{F}$ being the FFT operator and $\mathscr{F}^{-1}$ being the complex conjugate. In $(68), \quad \mathscr{F} \operatorname{div}\left(\mathbf{w}+\left(\beta_{1} / \gamma_{1}\right)\right)=\mathscr{F}\left((\nabla)^{1+}\right) \circ \mathscr{F}$ $\left(\mathbf{w}_{x}+\left(\beta_{1 \mathbf{x}} / \gamma_{1}\right)\right)+\mathscr{F}\left((\nabla)^{2+}\right) \circ \mathscr{F}\left(\mathbf{w}_{y}+\left(\beta_{1 \mathbf{y}} / \gamma_{1}\right)\right)$, where “。” denotes the componentwise multiplication and the division is also componentwise. 


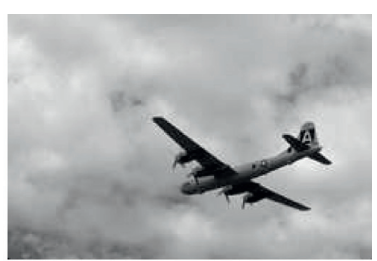

(a)

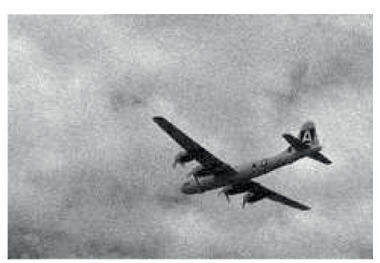

(e)

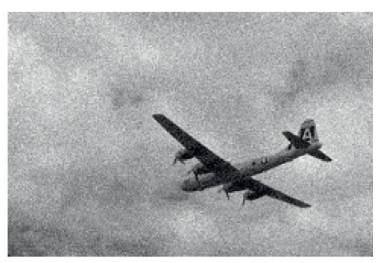

(i)

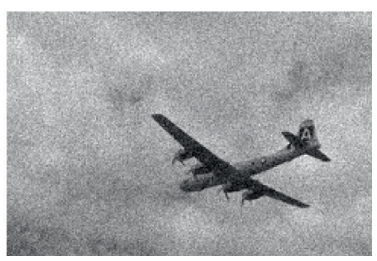

(m)

Original image

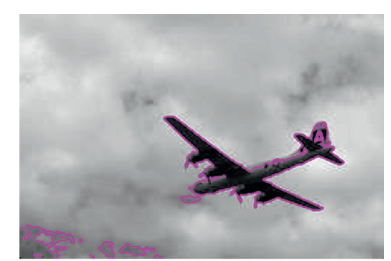

(b)

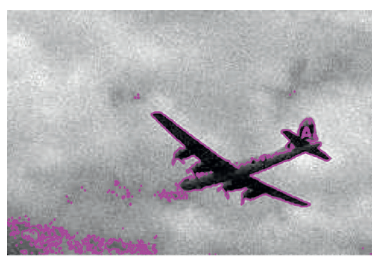

(f)

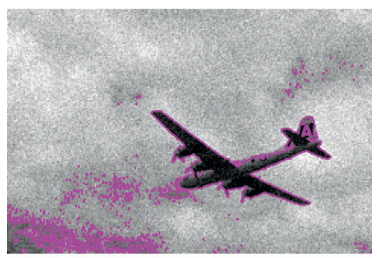

(j)

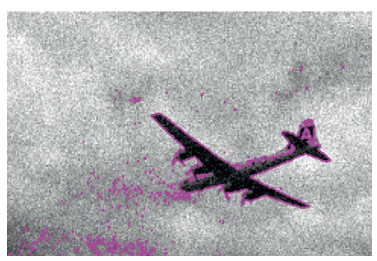

(n)

Chan - Vese method

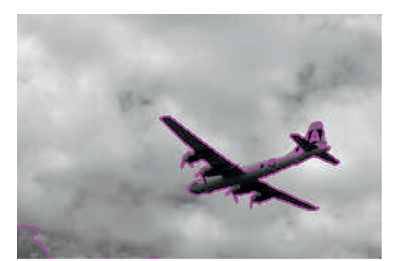

(c)

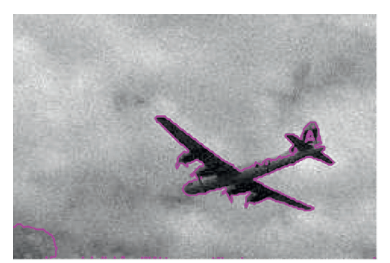

(g)

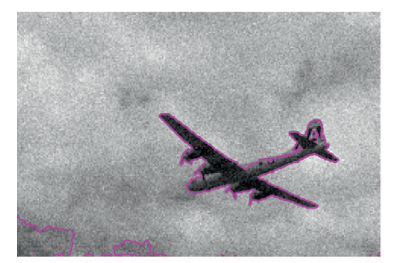

(k)

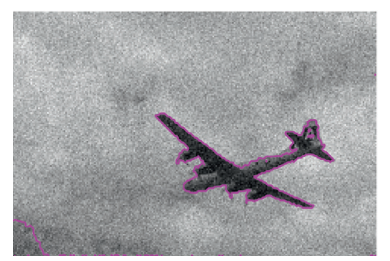

(o)

Bresson method

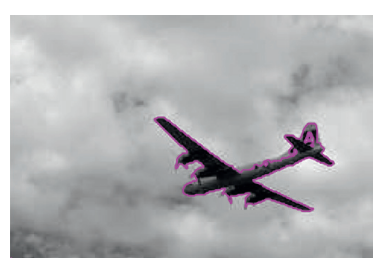

(d)

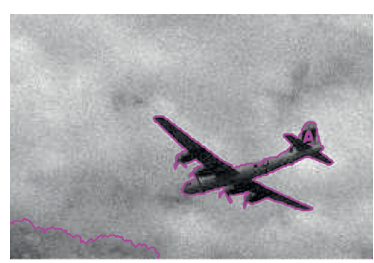

(h)

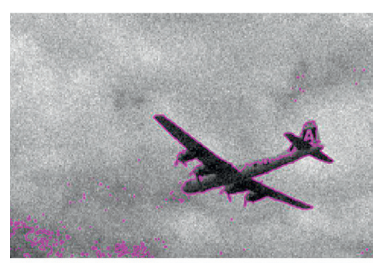

(1)

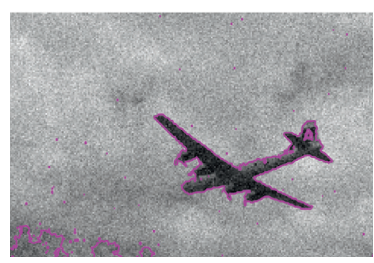

(p)

FIGURE 3: Illustration of without noisy natural image as well as three noisy image and the performance of all the three models by 3000 iterations. First row: the result of three methods for the original image segmentation without noise. Second row: the result of three methods for the original image segmentation with 0.1 noise. Third row: the result of three methods for the original image segmentation with 0.15 noise. Fourth row: the result of three methods for the original image segmentation with 0.2 noise.

\subsection{Algorithm for Solving (52). In this section, we solve} model (52) about $v$ :

$$
v:=\min _{v} \frac{1}{2 \theta}\|u-v\|_{2}^{2}+\lambda \int_{\Omega}\left(\left(I_{0}-c_{1}\right)^{2}-\left(I_{0}-c_{2}\right)^{2}\right) v+\alpha \nu(v) \mathrm{d} x
$$

The details are as follows: as introduced in (23) and since the constrained variable $v=u$, we calculate the minimization problem and get

$$
\theta \lambda\left(\left(I_{0}-c_{1}\right)^{2}-\left(I_{0}-c_{2}\right)^{2}\right)+(v-u)=0
$$

that is,

$$
v(x)=u(x)-\theta \lambda\left(\left(I_{0}-c_{1}\right)^{2}-\left(I_{0}-c_{2}\right)^{2}\right) .
$$

Thus, the $v$-minimization can be achieved through the following update: $v(x)=\min \left\{\max \left\{u(x)-\theta \lambda\left(\left(I_{0}-c_{1}\right)^{2}-\left(I_{0}-c_{2}\right)^{2}\right), 0\right\}, 1\right\}$.

Then, the framework of the algorithm which solves problem (52) can be illustrated as follows.

In this section, we give experimental justification of our proposed model and compare it with ACWE model [8] and Bresson method [24]. These experiments show that our method is robust in segmentation of objects in a range of images that have noise, have low contrast, and are directional. The proposed model was implemented by Matlab7 on a computer with Intel Core2 Duo2.2 GHz CPU, 2GB RAM, and Windows XP operating system. In numerical implementations, we only consider to use proposed model (52) for 


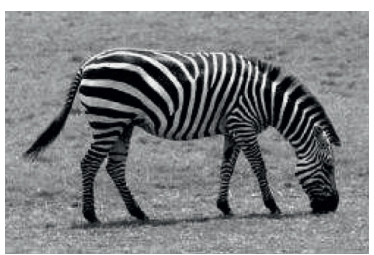

(a)

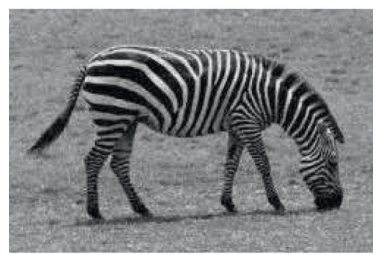

(e)

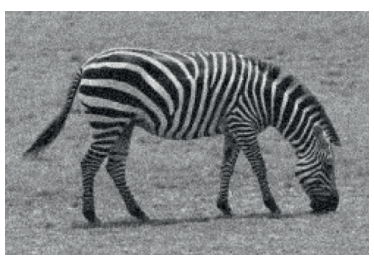

(i)

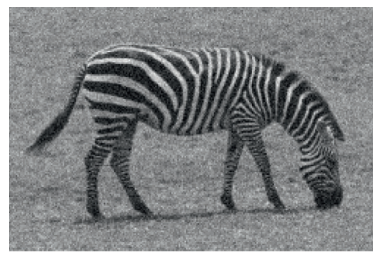

(m)

Original image

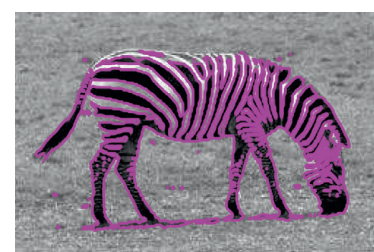

(b)

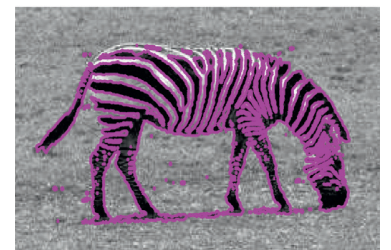

(f)

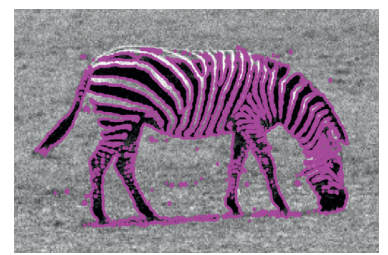

(j)

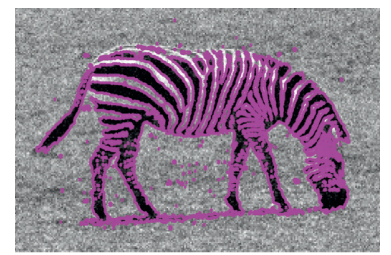

(n)

Chan - Vese method

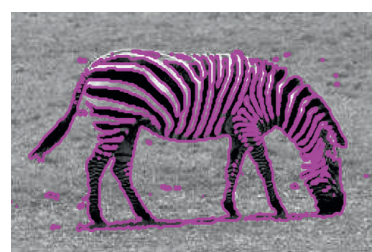

(c)

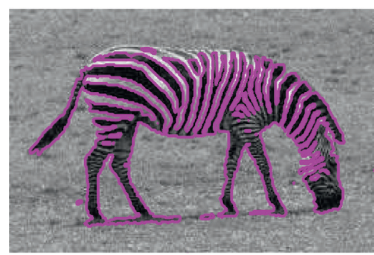

(g)

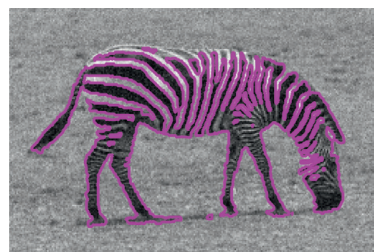

(k)

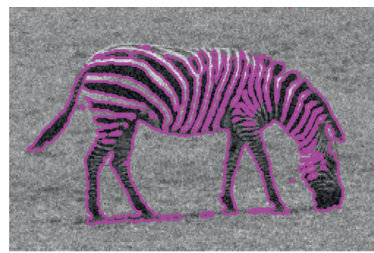

(o)

Bresson method

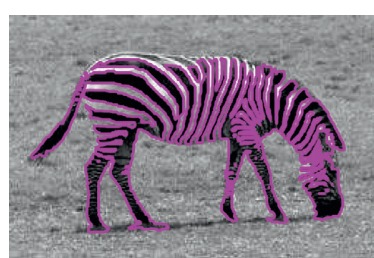

(d)

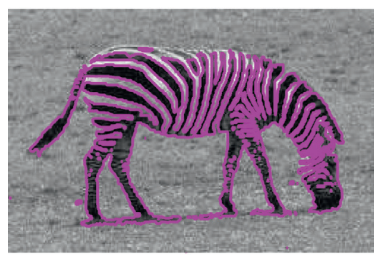

(h)

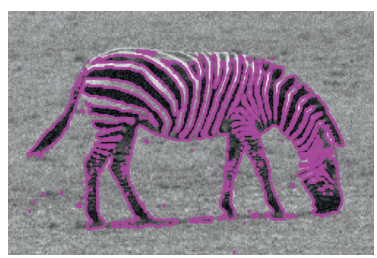

(1)

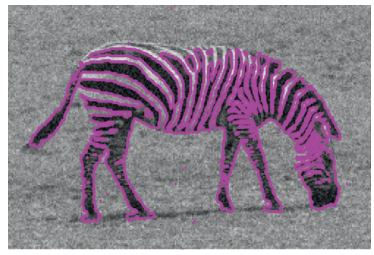

(p)

Our method

FIGURE 4: Illustration of without noisy natural image as well as three noisy image and the performance of all the three models by 3000 iterations. First row: the result of three methods for the original image segmentation without noise. Second row: the result of three methods for the original image segmentation with 0.1 noise. Third row: the result of three methods for the original image segmentation with 0.15 noise. Fourth row: the result of three methods for the original image segmentation with 0.2 noise.

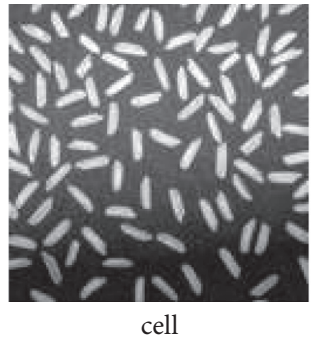

cell

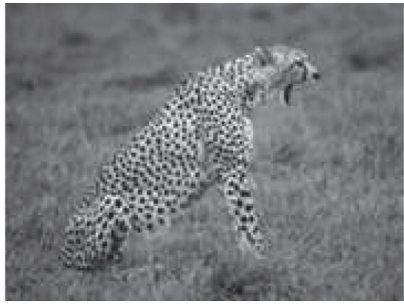

lion

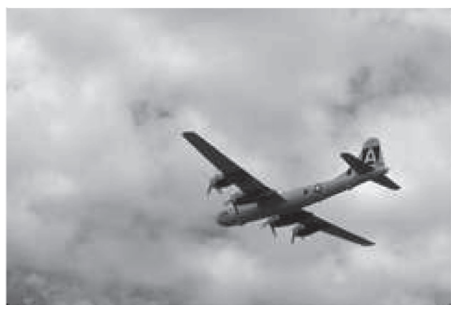

plane

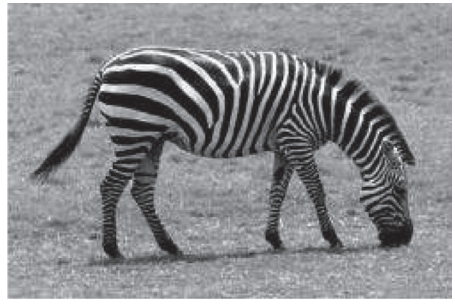

zebra

Figure 5: The given four original images.

the basic image segmentation problems. In fact, our model has been applied to synthetic and real images in this section.

3.3. Experimental Results of Real Images. In this section, the effectiveness of the segmentation models and algorithms is verified by some synthetic images. We first segment every original image into four images by adding $0.1,0.15$, and 0.2 random noise. The computer is Windows 7, a 64-bit operating system. The four original images with their three-level noise have been processed, and the results are illustrated in Figures $1-4$. Slight tuning of model parameters is necessary between images (but not between models for a single image).

Figure 5 gives four original images which will be segmented. Figures 1-4 show the segmentation results of the proposed method and the related methods. The first line of Figure 1 shows the segmentation of the original pictures, and the second and third lines are the segmentation results by adding 0.1 noise and 0.15 noise to the original image, respectively; we can see that our method can be used to 


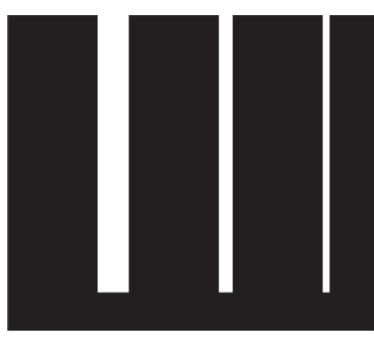

(a)

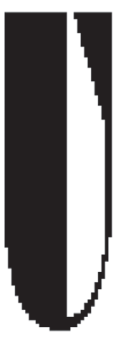

(b)

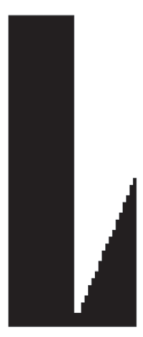

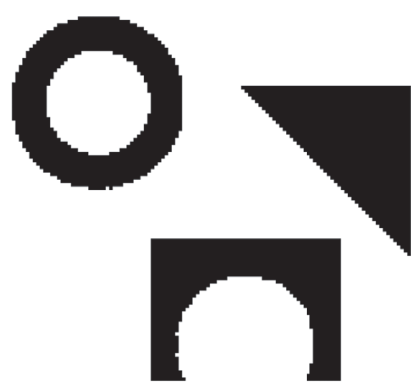

(c)

FIGURE 6: Sizes of the synthetic images is $128 \times 128$; the pixel values of these images are only 0.4 and 0.8 .

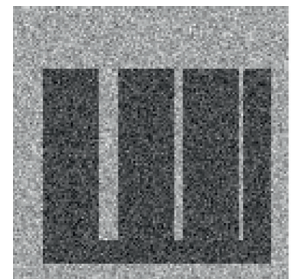

(a)

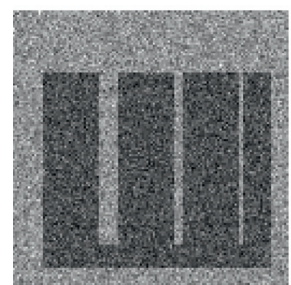

(e)

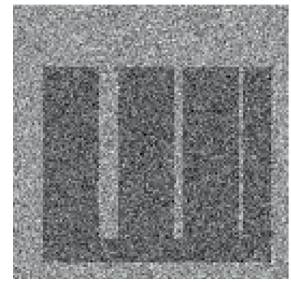

(i)

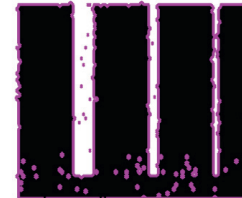

(b)

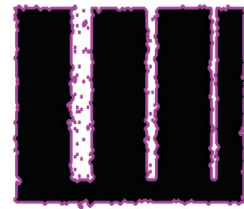

(f)

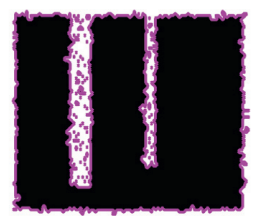

(j)

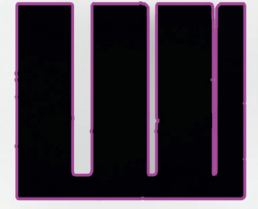

(c)

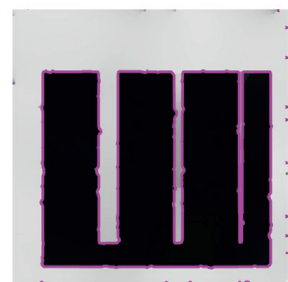

(g)

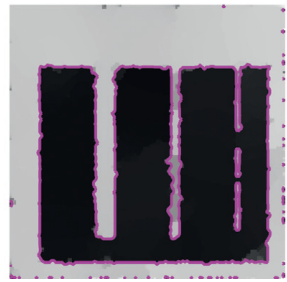

(k)

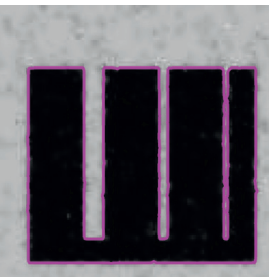

(d)

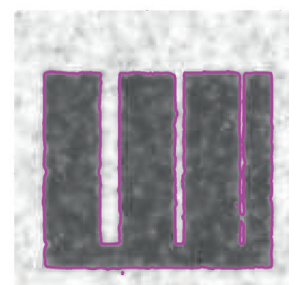

(h)

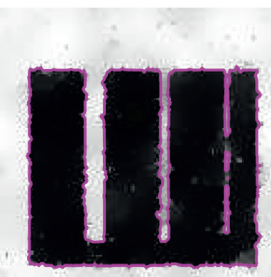

(1)

Chan - Vese method

Our method

Figure 7: Segmentation results to the synthetic image by using Bresson method [24], Chan-Vese [8], and our Algorithm 2 for different levels of noises. First row: the result of three methods for the original image segmentation with 0.1 noise. Second row: the result of three methods for the original image segmentation with 0.15 noise. Third row: the result of three methods for the original image segmentation with 0.2 noise.

successfully segment for the bottom part of the image, and the other two methods all failed; from the fourth line, although we have not fully segmented, we can see that our segmentation is more full at several locations below the image.

Figure 2 shows that our method is successful when the image has no noise and 0.1 random noise. When the image is added 0.15 random noise and 0.2 random noise, the $\mathrm{CV}$ method failed in the three methods, and compared with the Bresson method, we can see $(l)$ and $(p)$ provide more details in the lion's neck. In Figure 3, compared with the $\mathrm{CV}$ method, we have a more obvious advantage for the arc of the aircraft's tail and the head of the aircraft. And compared with the Bresson method, for the letter A on the tail, we are more clear. From Figure 4, we can see that our method is better.

3.4. Experimental Results of Synthetic Images. We give three synthetic images and add three different degrees of random noise in this part, and the effectiveness of the segmentation models and algorithms proposed in the following is verified by comparison with related models. We test four typical real images and tested each image with three different degrees of random noise. Figure 6 shows that the size of the synthetic 


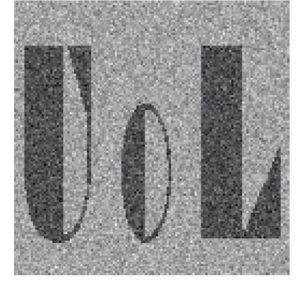

(a)

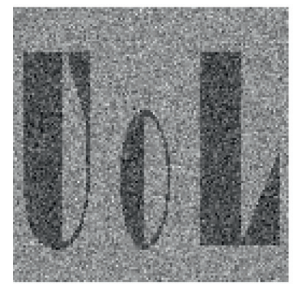

(e)

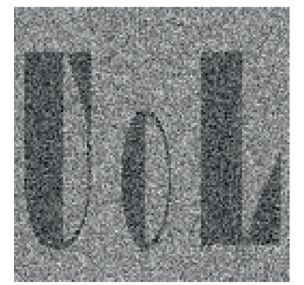

(i)

Original image

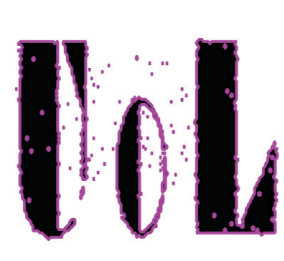

(b)

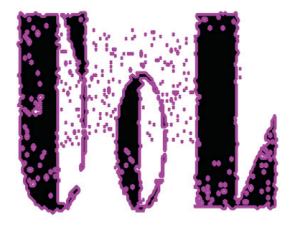

(f)

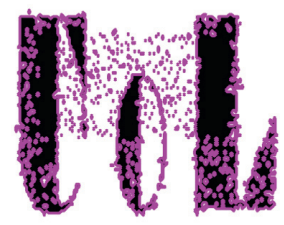

(j)

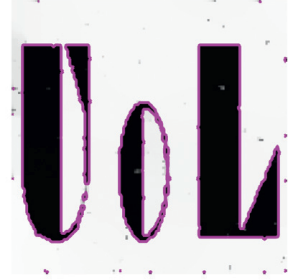

(c)

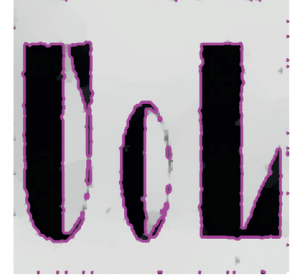

(g)

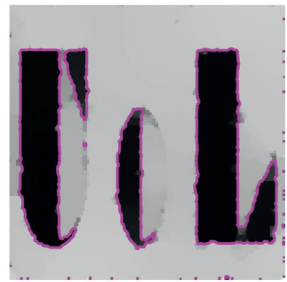

(k)

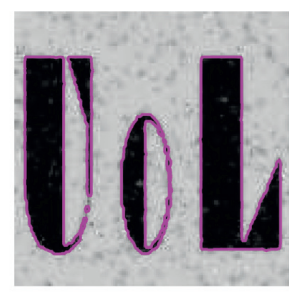

(d)

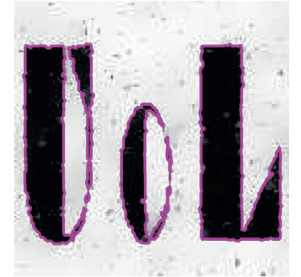

(h)

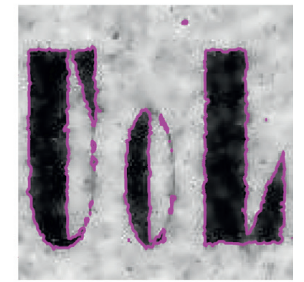

(l)

Our method

FIGURE 8: Segmentation results to the synthetic image by using Bresson method [24], Chan-Vese [8], and our Algorithm 2 for different levels of noises. First row: the result of three methods for the original image segmentation with 0.1 noise. Second row: the result of three methods for the original image segmentation with 0.15 noise. Third row: the result of three methods for the original image segmentation with 0.2 noise.

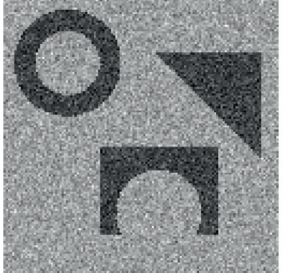

(a)

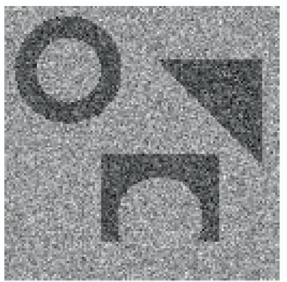

(e)

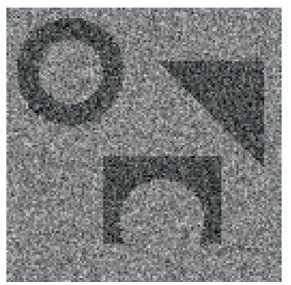

(i)

Original image

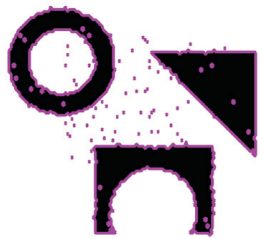

(b)

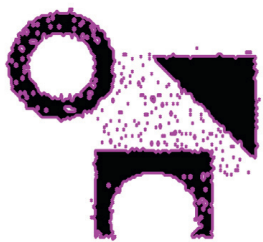

(f)

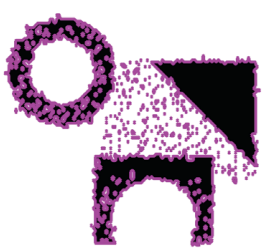

(j)

Chan - Vese method

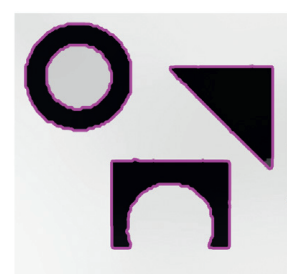

(c)

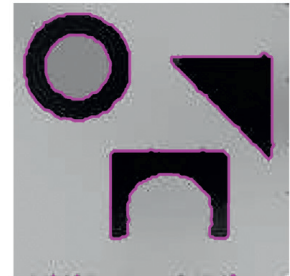

(g)

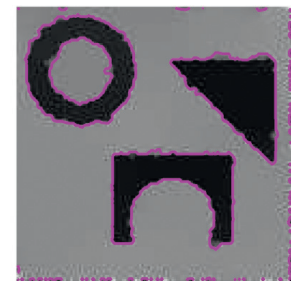

(k)

Bresson method

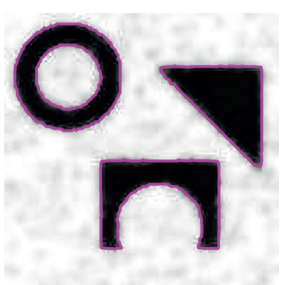

(d)

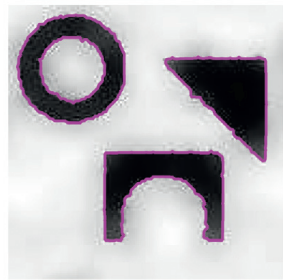

(h)

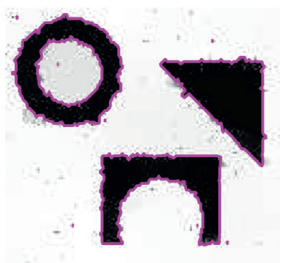

(1)

Our method

Figure 9: Segmentation results to the synthetic image by using Bresson method [24], Chan-Vese [8], and our Algorithm 2 for different levels of noises. First row: the result of three methods for the original image segmentation with 0.1 noise. Second row: the result of three methods for the original image segmentation with 0.15 noise. Third row: the result of three methods for the original image segmentation with 0.2 noise. 
Input $\theta, \gamma_{1}, \gamma_{1}>0$, set the starting values $\mathbf{w}=\mathbf{T}, \gamma_{1}=\gamma_{2}$, the constants $c_{1}$ and $c_{2}$ are updated periodically every 10 iterations, let $k=0$ and start $k$ - th iteration, which includes the following steps, until a stopping criterion $\max \left(\left|u_{n+1}-u_{n}\right|,\left|v_{n+1}-v_{n}\right|\right) \leq \varepsilon$ is satisfied:

(i) Update $\mathbf{T}$ by using $\mathbf{T}^{k+1}=\max \left\{\left\|G \mathbf{w}^{k}-\left(\beta_{2}^{\mathbf{k}} / \gamma_{2}\right)\right\|_{1}-\left(1 / \gamma_{2}\right), 0\right\}\left(\left(G \mathbf{w}^{k}-\left(\beta_{2}^{\mathbf{k}} / \gamma_{2}\right)\right) /\left\|G \mathbf{w}^{k}-\left(\beta_{2}^{\mathbf{k}} / \gamma_{2}\right)\right\|_{1}\right)$,

(ii) Update $\beta_{2}$ by using $\beta_{2}^{\mathbf{k}+1}=\beta_{2}^{\mathbf{k}}+\gamma_{2}\left(\mathbf{T}^{\mathbf{k}+1}-G \mathbf{w}^{\mathbf{k}}\right)$,

(iii) Update the $\mathbf{w}$, solving $\mathbf{w}^{k}$ by using $\mathbf{w}^{k+1}=\left(\gamma_{2} G\left(\mathbf{T}^{k+1}+\left(\beta_{2}^{\mathbf{k}+1} / \gamma_{2}\right)\right)+\gamma_{1}\left(u^{k}-\left(\beta_{1}^{\mathbf{k}} / \gamma_{1}\right)\right) /\left(\gamma_{1}+\gamma_{2} G^{2}\right)\right)$,

(iv) Update $\beta_{1}$ by using $\beta_{1}^{\mathbf{k}+1}=\beta_{1}^{\mathbf{k}}+\gamma_{1}\left(\mathbf{w}^{\mathbf{k}}-\nabla \mathbf{u}^{\mathbf{k}}\right)$,

(v) Update $u$ by using $u=\mathscr{F}^{-1}\left(\left(\mathscr{F}\left(v^{k}\right)-\theta \gamma_{1} \mathscr{F}(\operatorname{div})\left(\mathbf{w}^{k+1}+\left(\beta_{1}^{\mathbf{k}+1} / \gamma_{1}\right)\right)\right) /\left(1-\theta \gamma_{1} \mathscr{F}(\Delta)\right)\right)$,

(vi) Update $v$ by using $v=\min \left\{\max \left\{u^{k+1}-\theta \lambda\left(\left(I_{0}-c_{1}\right)^{2}-\left(I_{0}-c_{2}\right)^{2}\right), 0\right\}, 1\right\}$,

(vii) Let $k=k+1$ go to the $k+1$ iteration until converge.

Algorithm 2: Solving the model (52).

TABle 1: The err value in Figure 7 by using the Algorithm 2.

\begin{tabular}{lccc}
\hline & Image with 0.1 noise & Image with 0.15 noise & Image with 0.2 noise \\
\hline Chan - Vese method & 0.0085 & 0.0150 & 0.0356 \\
Bresson method & $8.5449 e-04$ & 0.0031 & 0.0143 \\
Our method & $\mathbf{7 . 3 2 4 2 e - 0 4}$ & $\mathbf{0 . 0 0 2 9}$ & $\mathbf{0 . 0 1 2 0}$ \\
\hline
\end{tabular}

Bold indicates that the err rate is the smallest compared with other two methods.

TABLE 2: The err value in Figure 8 through Algorithm 2.

\begin{tabular}{lccc}
\hline & Image with 0.1 noise & Image with 0.15 noise & Image with 0.2 noise \\
\hline Chan - Vese method & 0.0139 & 0.0331 & 0.0598 \\
Bresson method & 0.0031 & 0.0114 & 0.0199 \\
Our method & $\mathbf{0 . 0 0 1 4}$ & $\mathbf{0 . 0 0 9 3}$ & $\mathbf{0 . 0 1 9 0}$ \\
\hline
\end{tabular}

TAвLE 3: The err value in Figure 9 by Algorithm 2.

\begin{tabular}{lccc}
\hline & Image with 0.1 noise & Image with 0.15 noise & Image with 0.2 noise \\
\hline Chan - Vese method & 0.0090 & 0.0244 & 0.0422 \\
Bresson method & 0.0013 & 0.0045 & 0.0114 \\
Our method & $\mathbf{0 . 0 0 1 0}$ & $\mathbf{0 . 0 0 4 0}$ & $\mathbf{0 . 0 1 0 9}$ \\
\hline
\end{tabular}

The bold values illustrate that our method is the most effective since it is smallest.

images is $128 \times 128$ and the pixel values of these images are only 0.4 and 0.8 .

Before doing these experiments, we make some explanations as follows:

(1) For the above three synthetic images, we have two pixel values of 0.4 and 0.8 .

(2) We use the index error rate (err) to measure the similarity of the initial image and the segmentation, which are defined as follows, where $P$ represent the test image, $I$ represent the initial image:

$$
\operatorname{err}(P, I)=\frac{|P \neq I|}{|I|}
$$

From the definition, we can obtain that the closer the value of err is to 0 , the closer the image $P$ is to the image $I$ (Figures 7-9).

The error rate for Figure 7 by using Algorithm 2 is shown in Table 1.
The error rate for the synthetic image is shown in Table 2. The error rate for the synthetic image is shown in Table 3.

The closer the value of err is to 0 , the better the quality of the segmentation is. The err tests can be seen in Tables 1 to 3 where all the three models are tested on simple synthetic images, and these results validate quantitatively the outstanding performance of the Chan-Vese model and the Bresson method in comparison with the competing models.

\section{Discussion}

In this paper, we have proposed a new variational model suitable for segmentation a range of images with a blurred edge, a certain degree of noise, and a directional texture. $L_{2,1}$ norm is made for the boundary indication function, and different weights are taken for the $x$ direction and $y$ direction of the boundary at a certain place of the image. Experimental results on both real and synthetic images demonstrated that our method is very robust and efficient. We will further improve the proposed model to extract the colorful image in our future work. 


\section{Data Availability}

No data were used to support this study.

\section{Conflicts of Interest}

The authors declare that they have no conflicts of interest.

\section{Acknowledgments}

This work was supported by Henan Province Natural Science Foundation Project (212300 410320 ).

\section{References}

[1] N. Paragios and R. Deriche, "Geodesic active regions and level set methods for supervised texture segmentation," International Journal of Computer Vision, vol. 46, no. 3, pp. 223-247, 2002.

[2] Y. Wang, W. Yin, and Y. Zhang, "A fast algorithm for image deblurring with total variation regularization," Mathematics, 2007.

[3] Y. Hong, J. Yi, and D. Zhao, "Improved mean shift segmentation approach for natural images," Applied Mathematics and Computation, vol. 185, no. 2, pp. 940-952, 2007.

[4] R. W. Liu, L. Shi, W. Huang, J. Xu, S. C. H. Yu, and D. Wang, "Generalized total variation-based MRI Rician denoising model with spatially adaptive regularization parameters," Magnetic Resonance Imaging, vol. 32, no. 6, pp. 702-720, 2014.

[5] W. Liu, J. Duan, Z. Qiu, Z. Pan, R. Wen Liu, and L. Bai, "Implementation of high-order variational models made easy for image processing," Mathematical Methods in the Applied Sciences, vol. 39, no. 14, pp. 4208-4233, 2016.

[6] Y. Guo, Y. Lu, and R. Wen Liu, "Lightweight deep networkenabled real-time low-visibility enhancement for promoting vessel detection in maritime video surveillance," Journal of Navigation, vol. 2021, pp. 1-21, 2021.

[7] D. Mumford and J. Shah, "Optimal approximations by piecewise smooth functions and associated variational problems," Communications on Pure and Applied Mathematics, vol. 42, no. 5, pp. 577-685, 1989.

[8] T. F. Chan and L. A. Vese, "Active contours without edges," IEEE Transactions on Image Processing, vol. 10, no. 2, pp. 266-277, 2001.

[9] S. Song Chun Zhu and A. Yuille, "Region competition: unifying snakes, region growing, and Bayes/MDL for multiband image segmentation," IEEE Transactions on Pattern Analysis and Machine Intelligence, vol. 18, no. 9, pp. 884-900, 1996.

[10] O. J. Tobias and R. Rui Seara, "Image segmentation by histogram thresholding using fuzzy sets," IEEE Transactions on Image Processing, vol. 11, no. 12, pp. 1457-1465, 2002.

[11] D. H. Zhu, "Threshold-based segmentation for 3D medical volumetric images," Computer Science, vol. 40, no. 1, pp. 269-272, 2013.

[12] S. R. Mcwilliams, O. J. O'Connor, A. M. McGarrigle et al., "CT-based estimation of intracavitary gas volumes using threshold-based segmentation: in vitro study to determine the optimal threshold range," Journal of Medical Imaging and Radiation Oncology, vol. 56, no. 3, pp. 289-294, 2012.

[13] A. Xu, L. Wang, S. Feng, and Y. Qu, "Threshold-based level set method of image segmentation," in Proceedings of the International Conference on Intelligent Networks and Intelligent Systems, pp. 703-706, IEEE, Shenyang, China, November 2011.
[14] T. F. Chan, S. Esedoglu, and M. Nikolova, "Algorithms for finding global minimizers of image segmentation and denoising models," SIAM Journal on Applied Mathematics, vol. 66, no. 5, pp. 1632-1648, 2006.

[15] V. Caselles, R. Kimmel, and G. Sapiro, "Geodesic active contours," International Journal of Computer Vision, vol. 22, no. 1, pp. 61-79, 1997.

[16] M. Kass, A. Witkin, and D. Terzopoulos, "Snakes: active contour models," International Journal of Computer Vision, vol. 1, no. 4, pp. 321-331, 1988.

[17] R. Goldenberg, R. Kimmel, E. Rivlin, and M. Rudzsky, "Fast geodesic active contours," IEEE Transactions on Image Processing, vol. 10, no. 10, pp. 1467-1475, 2001.

[18] S. Kichenassamy, A. Kumar, P. Olver, A. Tannenbaum, and A. Yezzi, "Gradient flows and geometric active contour models," in Proceedings of the IEEE International Conference on Computer Vision, pp. 810-815, Cambridge, MA, USA, June 1995.

[19] L. A. Vese and T. F. Chan, "A multiphase level set framework for image segmentation using the Mumford and Shah model," International Journal of Computer Vision, vol. 50, no. 3, pp. 271-293, 2002.

[20] A. Tsai, A. Yezzi, and A. S. Willsky, "Curve evolution implementation of the Mumford-Shah functional for image segmentation, denoising, interpolation, and magnification," IEEE Transactions on Image Processing, vol. 10, no. 8, pp. 1169-1186, 2001.

[21] M. A. Femina and S. P. Raajagopalan, "Anatomical structure segmentation from early fetal ultrasound sequences using global pollination CAT swarm optimizer-based Chan-Vese model," Medical, \& Biological Engineering \& Computing, vol. 57, no. 8, pp. 1763-1782, 2019.

[22] D. Jeong, S. Kim, C. Lee, and J. Kim, "An accurate and practical explicit hybrid method for the chan-vese image segmentation model," Mathematics, vol. 8, no. 7, p. 1173, 2020.

[23] T. Chan and L. Vese, "A multiphase level set framework for image segmentation using the Mumford and Shah model," International Journal of Computer Vision, vol. 50, no. 3, pp. 271-293, 2002.

[24] X. Bresson, S. Esedoḡlu, P. Vandergheynst, J.-P. Thiran, and S. Osher, "Fast global minimization of the active contour/ snake model," Journal of Mathematical Imaging and Vision, vol. 28, no. 2, pp. 151-167, 2007.

[25] H. Wang, T.-Z. Huang, and Y.-Q. Du, “A global minimization hybrid active contour model with applications to oil spill images," Computers \& Mathematics with Applications, vol. 68, no. 3, pp. 353-362, 2014.

[26] H. Xu, G. Jiang, M. Yu, and T. Luo, “A global and local active contour model based on dual algorithm for image segmentation," Computers \& Mathematics with Applications, vol. 74, no. 6, pp. 1471-1488, 2017.

[27] A. Chambolle, "An algorithm for total variation minimization and applications," Journal of Mathematical Imaging and Vision, vol. 20, no. 1-2, pp. 89-97, 2004.

[28] J.-F. Aujol, G. Gilboa, T. Chan, and S. Osher, "Structuretexture image decomposition-modeling, algorithms, and parameter selection," International Journal of Computer Vision, vol. 67, no. 1, pp. 111-136, 2006.

[29] H. H. Bauschke and P. L. Combettes, Convex Analysis and Monotone Operator Theory in Hilbert Spaces, Springer, Berlin, Germany, 2010.

[30] D. P. Bertsekas, Convex Analysis and Optimization, Athena Scientific, Nashua, NH, USA, 2003. 
[31] J.-F. Aujol, "Some first-order algorithms for total variation based image restoration," Journal of Mathematical Imaging and Vision, vol. 34, no. 3, pp. 307-327, 2009.

[32] C. Wu and X.-C. Tai, "Augmented Lagrangian method, dual methods, and split bregman iteration for ROF, vectorial TV, and high order models," SIAM Journal on Imaging Sciences, vol. 3 , no. 3, pp. 300-339, 2010.

[33] J. J. Moreau, "Fonctiones convexes duales et points proximaux dans un espace hilbertien," Computes Rendus de lAcadmie des Sciences de Paris, vol. 255, pp. 2897-2899, 1962.

[34] P. L. Combettes and V. R. Wajs, "Signal recovery by proximal forward-backward splitting," Multiscale Modeling and Simulation, vol. 4, no. 4, pp. 1168-1200, 2005.

[35] C. Liu, F. Dong, S. Zhu, D. Kong, and K. Liu, "New variational formulations for level set evolution without reinitialization with applications to image segmentation," Journal of Mathematical Imaging and Vision, vol. 41, no. 3, pp. 194-209, 2011. 